\title{
Photoexcited carrier relaxation dynamics in pentacene probed by ultrafast optical spectroscopy: Influence of morphology on relaxation processes
}

\author{
V.K. Thorsmølle a,b,*, R.D. Averitt ${ }^{\text {a,c }}$, J. Demsar ${ }^{\text {a,d,e }}$, D.L. Smith ${ }^{\text {a }}$, S. Tretiak ${ }^{\text {a }}$, R.L. Martin ${ }^{\text {a }}$, \\ X. Chi ${ }^{\text {a,f }}$, B.K. Crone ${ }^{\text {a }}$, A.P. Ramirez ${ }^{\text {a,g }}$, A.J. Taylor ${ }^{a}$ \\ a Los Alamos National Laboratory, Los Alamos, NM 87545, USA \\ ${ }^{\mathrm{b}}$ Ecole Polytechnique Fédérale de Lausanne, CH-1015 Lausanne, Switzerland \\ c Boston University, Boston, MA 02215, USA \\ d Department of Physics and Center for Applied Photonics, Universität Konstanz, D-78457 Konstanz, Germany \\ e Department of Complex Matter, Jozef Stefan Institute, Ljubljana, Slovenia \\ ${ }^{\mathrm{f}}$ Texas AEM University-Kingsville, Kingsville, TX 78363, USA \\ ${ }^{\mathrm{g}}$ Bell Laboratories, Alcatel-Lucent, 600 Mountain Avenue, Murray Hill, NJ 07974, USA
}

\section{A R T I C L E I N F O}

PACS:

71.35. $-\mathrm{y}$

74.62.Fj

73.61.Ph

78.47.-p

Keywords:

Ultrafast spectroscopy

Organic semiconductors

Sample morphology

\begin{abstract}
A B S T R A C T
We present a comparative study of ultrafast photoexcited state relaxation in pentacene single crystals and in pure and $\mathrm{C}_{60}$-doped pentacene films using optical pump-probe spectroscopy. The photoinduced absorption spectra in pentacene crystals is consistent with a dominant singlet-triplet fission decay channel for above-gap excitation. This decay channel is suppressed in thin films and even further suppressed by electron trapping in $\mathrm{C}_{60}$-doped films. Thus we show that suppression of triplet state production, which is necessary for free carrier formation and thus photovoltaic and photodiode performance, is controllable via sample morphology.
\end{abstract}

(c) 2009 Elsevier B.V. All rights reserved.

\section{Introduction}

The strong potential for the use of organic semiconductors in technological applications as an alternative to inorganic semiconductors has in recent years spurred a tremendous research effort in such materials [1-3]. Applications include thin-film transistors [4], light-emitting diodes [5,6], lasers and solar cells $[7,8]$. In order to successfully implement organic materials in these technologies it is vital to understand the photoconductive properties as well as the nature of the photogenerated states including their relaxation dynamics. Furthermore, it is important to understand the nature of defects and impurities, the presence of which may obscure the study of intrinsic properties and inhibit device performance $[9,10]$.

Exposure of an organic semiconductor to light above the absorption edge gives rise to various photoexcited species. There is an ongoing debate in the literature as to the nature of these photoexcitations and their relaxation dynamics. This

\footnotetext{
* Corresponding author at: Ecole Polytechnique Fédérale de Lausanne, CH-1015 Lausanne, Switzerland. Tel.: +4121693 3188; fax: +41216934111.

E-mail address: verner.thorsmolle@epfl.ch (V.K. Thorsmølle).
}

includes details of exciton formation versus the generation of charged polarons or free charge carriers [11-18]. These processes are important in determining the behavior of many organic semiconductor-based devices such as solar cells and photodiodes. Organic polymers, such as poly( $p$-phenylene-vinylene), have been extensively studied providing observations of polarons, interchain excitons (indirect excitons or bound polaron pairs), self-trapped excitons, triplet excitons and charge transfer dynamics [19-24]. In contrast to organic polymers with long chains, organic molecular crystals consist of shorter molecular units and form well-defined crystal structures. The intermolecular interactions are weak, and the excitons are largely confined to single molecules resulting in large exciton binding energies [25]. Polyacene organic crystals such as pentacene (Pc) are model systems for studying the intrinsic properties of exciton dynamics.

Pc molecules consist of five benzene rings fused along their sides and arranged in a herringbone stacking arrangement with two molecules in each unit cell [2]. Pentacene is opaque and is nonluminescent [26]. In the polyacene series of organic crystals, the energy level of the lowest triplet exciton $E\left(\mathrm{~T}_{1}\right)$ decreases faster than the lowest singlet exciton energy $E\left(S_{1}\right)$ with increasing molecular size. The energy difference $E\left(\mathrm{~S}_{1}\right)-2 E\left(\mathrm{~T}_{1}\right)$ is $-1.3 \mathrm{eV}$ in napthalene $(\mathrm{Nph}),-0.55 \mathrm{eV}$ in anthracene $(\mathrm{Ac}),-0.21 \mathrm{eV}$ in 
tetracene (Tc), and $0.11 \mathrm{eV}$ in Pc [26]. In Pc, the excitonic fission process from the lowest singlet exciton to a pair of the lowest triplet excitons $S_{1} \rightarrow 2 T_{1}$ is energetically allowed, while in Tc this same process is only possible by thermal activation. This process is strongly suppressed in Nph and Ac. An energy level diagram for Pc is presented in Fig. 1. Direct fission from higher-lying singlet states, $\mathrm{S}_{N} \rightarrow 2 \mathrm{~T}_{1}$, is another probable relaxation channel which competes with nonradiative relaxation to the lowest excited state, $S_{1}$ [1]. Charge transfer processes, resulting in carrier generation, compete with both singlet and triplet exciton formation. In some device applications, such as solar cells and photodiodes it is beneficial to enhance carrier generation.

In this paper we present a comparative study of the ultrafast dynamics in Pc single crystals and films using optical pump-probe spectroscopy. In particular, we excite the samples with above gap optical pulses and study the induced changes in optical properties over a wide range of photon energies (from 0.6 to $2.5 \mathrm{eV}$ ) aiming to follow various relaxation channels as depicted in Fig. 1. We show that in Pc single crystals the dynamics is dominated by singlet-triplet fission. However, in undoped and $\mathrm{C}_{60}$-doped Pc films, the triplet production is quenched, suggesting that the presence of defects (electron acceptors) results in competing charge transfer dynamics.

\section{Results and discussion}

The Pc crystals used were grown in a flow of inert gas. They were typically $3 \times 3 \mathrm{~mm}^{2}$ and approximately $50 \mu \mathrm{m}$ thick. The Pc films were evaporated onto $10 \times 10 \mathrm{~mm}^{2} \mathrm{MgO}$ substrates, with a film thickness of $\sim 150 \mathrm{~nm}$. One of the films was doped with $\sim 0.03 \%$ of molecular $\mathrm{C}_{60}$.

In these experiments we utilized a commercial-based regeneratively amplified $\mathrm{Ti}: \mathrm{Al}_{2} \mathrm{O}_{3}$ laser system operating at $250 \mathrm{kHz}$ producing nominally $10 \mu \mathrm{J}$, sub-50 fs pulses at $1.5 \mathrm{eV}$. The samples were excited at $3.0 \mathrm{eV}$ (high above the absorption band of $\sim 1.9 \mathrm{eV}$ in Pc) with an excitation fluence of up to $\sim 150 \mu \mathrm{J} / \mathrm{cm}^{2}$, and the photoinduced (PI) changes in reflectivity $\Delta R / R$ (crystals) and transmissivity $\Delta T / T$ (films) were measured over the range of probe photon energies from 0.6 to $2.5 \mathrm{eV}$ using an optical parametric amplifier. The probe pulse polarization was optimized for maximum signal along the long molecular axis.

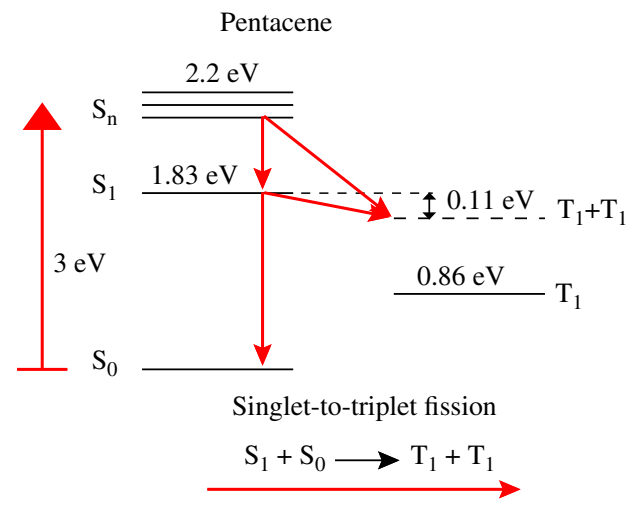

Fig. 1. Upper picture: Energy level diagram of Pc showing only the levels participating in the singlet-triplet fission processes. Here, $S_{N}$ represents higher lying states in the singlet manifold, $S_{1}$ the lowest singlet exciton, and $T_{1}$ the lowest triplet exciton. $T_{1}+T_{1}$ denotes two triplet excitons produced by fission. The fission processes, $\mathrm{S}_{1} \rightarrow 2 \mathrm{~T}_{1}, \mathrm{~S}_{N} \rightarrow 2 \mathrm{~T}_{1}$ from the singlet to the triplet manifold are denoted by arrows. Lower picture: The singlet-triplet fission process. In the singlet-triplet fission process, a singlet exciton, such as $S_{1}$, together with an unexcited molecule $\mathrm{S}_{0}$ transform into two triplet excitons $\mathrm{T}_{1}$, e.g. $\mathrm{S}_{1}+\mathrm{S}_{0} \rightarrow \mathrm{T}_{1}+\mathrm{T}_{1}$. The singlet-triplet fission process conserves spin.

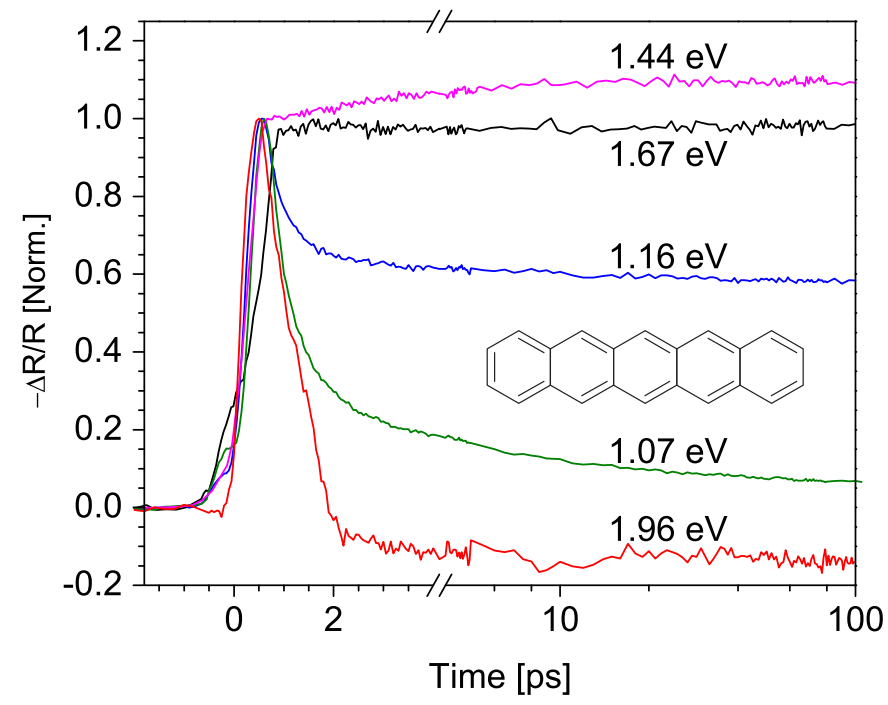

Fig. 2. Time-resolved PI reflectivity dynamics in Pc single crystal recorded at selected probe photon energies. The data are normalized to the value recorded after the initial sub-ps rise. The inset shows the molecular structure of Pc.

Fig. 2 presents the induced reflectivity traces recorded on Pc single crystal at selected probe photon energies following photoexcitation with $50 \mathrm{fs}$ optical pulses with a $3 \mathrm{eV}$ photon energy. The sub-ps rise is followed by an initial ps decay process after which a quasi-equilibrium is reached with a decay much longer than $1 \mathrm{~ns}$. This long-lived photoinduced absorption (PIA) is centered at $\sim 1.4 \mathrm{eV}$. The recovery of the induced change in reflectivity (transmission) at low and high photon energies with a $\sim 1$-ps exponential decay can be associated with $S_{1} \rightarrow S_{N}$ optical transitions. The internal conversion from $S_{N}$ to $S_{1}$ is reflected in the $1.07 \mathrm{eV}$ data with an exponential decay with a $\sim 0.55 \mathrm{ps}$ time constant.

The transient PI spectrum of the Pc crystal is shown in Fig. 3(a) at different times after photoexcitation. The most prominent feature is a long-lived ( $\gg 1 \mathrm{~ns}$ ) PIA peak centered at approximately $\sim 1.4 \mathrm{eV}$. The long relaxation time and the characteristic energy scale suggests that the state being probed is the triplet state $T_{1}$ $\left(\mathrm{T}_{1} \rightarrow \mathrm{T}_{N}\right)$, i.e. the PIA from the occupied $\mathrm{T}_{1}$ level to a higher-lying unoccupied excited state $\mathrm{T}_{N}$ [27].

The attribution of the long-lived PIA to the triplet production in Pc crystals is substantiated by probing the temperature dependence of the $\mathrm{T}_{1} \rightarrow \mathrm{T}_{N}$ transition with a probe energy of $1.44 \mathrm{eV}$. No temperature dependence was observed. This is expected, since $E\left(\mathrm{~S}_{1}\right)>2 E\left(\mathrm{~T}_{1}\right)$ and $E\left(\mathrm{~S}_{N}\right)>2 E\left(\mathrm{~T}_{1}\right)$. Furthermore, in the same measurements on Tc single crystals [28], where similar to Pc a longlived PIA peaked at $1.7 \mathrm{eV}$ is observed, a pronounced temperature dependence of this PIA is observed. In Tc, the PIA is enhanced at high temperatures, with its temperature dependence following a thermally activated behavior, in accordance with the triplet production scenario for polyacene crystals and the energy level diagram for Tc [28].

The initial ultrafast dynamics observed on a sub-ps time scale involves both $S_{N} \rightarrow 2 T_{1}$ fission and $S_{N} \rightarrow S_{1}$ internal conversion in the singlet manifold. The time scale of the $\mathrm{S}_{N} \rightarrow 2 \mathrm{~T}_{1}$ fission is $\sim 0.7 \mathrm{ps}$ and is temperature independent. Assuming an efficient triplet production in Pc crystals (neglecting charge generation [29]), an initial singlet-exciton concentration of $2.2 \times 10^{17} \mathrm{~cm}^{-3}$ (calculated from the excitation fluence) yields close to $4.4 \times 10^{17} \mathrm{~cm}^{-3}$ triplets.

The transient PI spectrum of Pc films, shown in Fig. 3(a), resembles a previously obtained spectrum published in Ref. [29], 


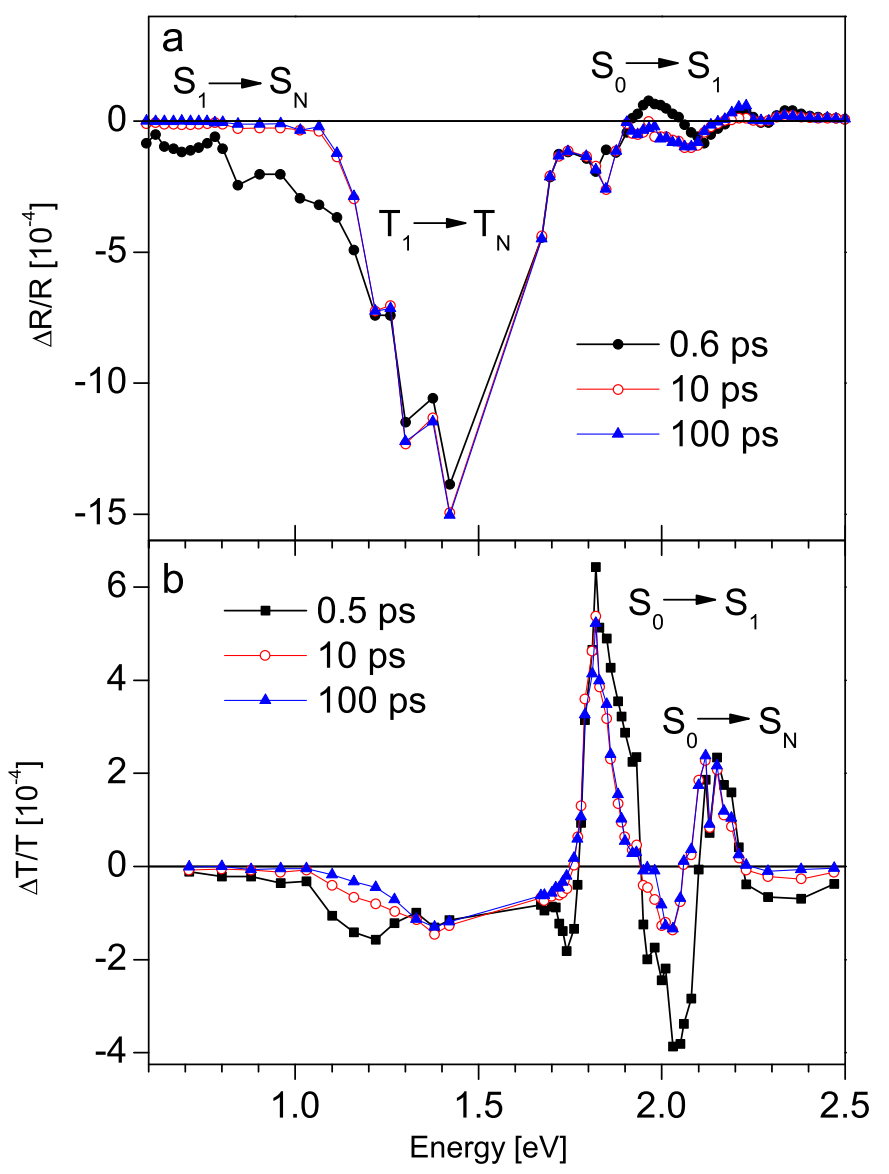

Fig. 3. (a) Transient spectra $\Delta R / R$ in Pc crystal at different pump-probe delay times. $\mathrm{S}_{0} \rightarrow \mathrm{S}_{1}$ and $\mathrm{S}_{N} \rightarrow \mathrm{S}_{1}\left(\mathrm{~T}_{1} \rightarrow \mathrm{T}_{N}\right)$ refer to electronic transitions in the singlet (triplet) manifold. (See Fig. 1.) (b) Photoinduced transmission spectra of Pc thin film at several time delays. All data were recorded at room temperature with $3.0 \mathrm{eV}$ optical pulses used for photoexcitation.

and the steady state absorption spectrum (not shown) is identical to the one given in Ref. [13]. In contrast to Pc crystals, the PI spectrum in Pc films is dominated by sharp features at higher energies, while the long-lived PIA centered at $\sim 1.4 \mathrm{eV}$ is substantially reduced. At room temperature we observe PI bleaching peaks at $\sim 1.8$ and $\sim 2.1 \mathrm{eV}$, and a PIA peak at $\sim 2.0 \mathrm{eV}$. Their decay dynamics consists of two contributions; a fast exponential $\sim 0.4$ ps decay followed by a sub-ns decay. The $\sim 1.8$ and $\sim 2.1 \mathrm{eV}$ are assigned to $\mathrm{S}_{0} \rightarrow \mathrm{S}_{1}$ and $\mathrm{S}_{0} \rightarrow \mathrm{S}_{N}$ transitions, respectively. With lowering the temperature, the smaller $2.0 \mathrm{eV}$ PIA peak transforms into a narrower and substantially larger PIA peak at $1.9 \mathrm{eV}$ (see Fig. 4). This PIA peak at $\sim 1.9 \mathrm{eV}$ persists well into the ns regime. This strongly temperature-dependent PIA observed at $\sim 1.9 \mathrm{eV}$ is attributed to $\mathrm{S}_{0}^{+} \rightarrow \mathrm{S}_{1}^{+}$transitions, and is related to charge transfer dynamics. $\left(\mathrm{S}_{0}^{+} \rightarrow \mathrm{S}_{1}^{+}\right.$refers to an ionized state, where an electron from the Pc molecule has been transferred to an electron trap.)

Importantly, the same dynamics and spectral features as in films are observed also in the $\mathrm{C}_{60}$-doped Pc film as shown in Fig. 4. The main difference between the two is that the spectral features above $\sim 1.7 \mathrm{eV}$ are in $\mathrm{C}_{60}$-doped Pc film enhanced by $\sim 1.5$ times, while the PIA at $\sim 1.4 \mathrm{eV}$ is further suppressed. With $\mathrm{C}_{60}$ being a known electron acceptor, this observation further lends support to the assignment of the $\sim 1.9 \mathrm{eV}$ absorption peak to charge transfer due to electron traps intrinsic to Pc films. The trapping of electrons breaks apart excitons, leaving behind free holes, and causes a charge-separated state to be formed [30].

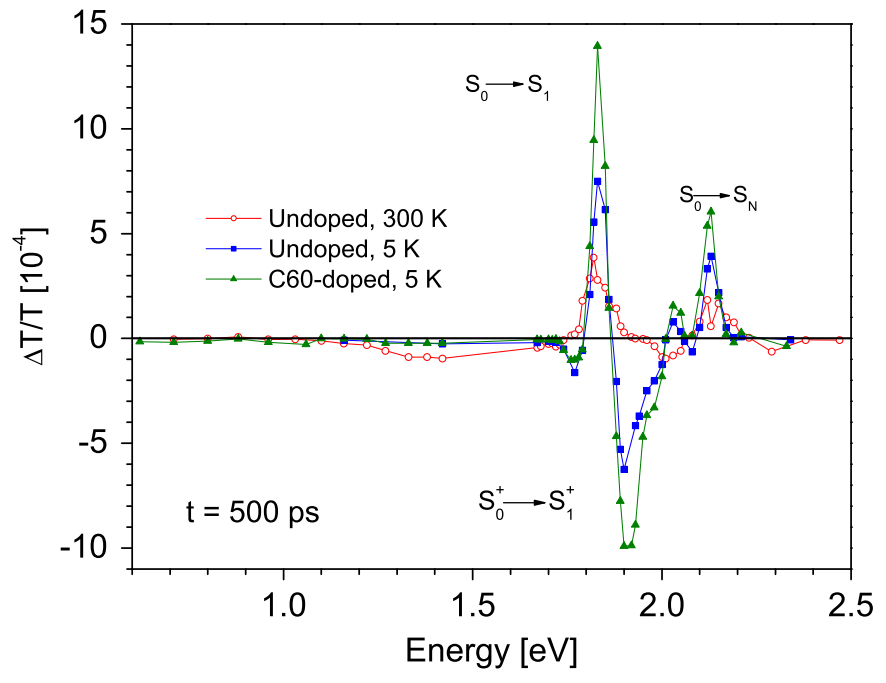

Fig. 4. Comparison of $\Delta T / T$ spectra recorded in undoped and $C_{60}$-doped Pc films at $500 \mathrm{ps}$ after photoexcitation. $\mathrm{S}_{0}^{+} \rightarrow \mathrm{S}_{1}^{+}$refers to an ionized state at $1.9 \mathrm{eV}$ due to electron transfer.

The long-lived $\mathrm{T}_{1} \rightarrow \mathrm{T}_{N}$ transition at $\sim 1.4 \mathrm{eV}$, clearly prominent in the Pc crystal, is strongly suppressed in the Pc film, Fig. 3(b), where only a minor feature with sub-ns dynamics is observed which vanishes at low temperatures. Considering the identical dynamics, temperature dependence and an enhancement of the spectral features in the $\mathrm{C}_{60}$-doped $\mathrm{Pc}$ film as compared to the undoped Pc film, we attribute the quenching of the long-lived PIA at $\sim 1.4 \mathrm{eV}$ to be due to ultrafast charge transfer dynamics related to electron traps in the Pc films.

\section{Conclusions}

In addressing the importance of the ultrafast processes we find singlet-triplet fission being the dominant process in Pc crystals with the display of a prominent long-lived PIA peak due to photogenerated triplet excitons created via $\mathrm{S}_{1} \rightarrow 2 \mathrm{~T}_{1}$ and $\mathrm{S}_{N} \rightarrow 2 \mathrm{~T}_{1}$ fission. Triplet exciton production is found to be independent of the lattice temperature, in contrast to Tc where it is strongly temperature dependent in agreement with the thermally activated $S_{1} \rightarrow 2 T_{1}$ fission process [28]. In comparison to Pc crystals, we find that in Pc films the triplet production is quenched and the dynamics is largely dominated by charge transfer processes originating from defects (electron acceptors). This assignment was further supported by measurements on $\mathrm{C}_{60}$-doped Pc films, where further suppression of the long-lived PIA centered at $1.4 \mathrm{eV}$ is observed. With $\mathrm{C}_{60}$ being a known electron acceptor, these data lend further support to association of observed changes in the photoinduced spectral shape and its dynamics in films (as compared to crystals) to charge transfer processes.

We have shown that defects can cause triplet quenching and enhanced carrier generation. Recent studies have shown that even the best single crystals used in FETs based on these smallmolecule systems have a large density of defects [31]. Depending on the device application, it may be desirable to either enhance or suppress carrier generation. This suggests that especially for power and light generation, organic heterostructures will need to be composed of controlled defect-concentration material. The results of Pc crystals and films demonstrate the sensitivity of exciton dynamics to defects. Importantly, we have demonstrated how sample morphology may control relaxation behavior 
and charge transport in an organic semiconductor by effectively turning fission decay channels on and off.

\section{Acknowledgments}

This work was supported by the Laboratory Directed Research and Development program at Los Alamos National Laboratory, the Department of Energy (DOE) Center for Integrated Nanotechnologies, and DOE Grant no. DE-FG02-04ER46118. We are grateful to Michael Grätzel, Majed Chergui, Christoph Gadermaier, Thomas Dekorsy and Alfred Leitenstorfer for valuable comments.

\section{References}

[1] M. Pope, C.E. Swenberg, Electronic Processes in Organic Crystals and Polymers, second ed., Oxford University Press, New York, 1999.

[2] F.A. Hegmann, Phys. Canada 59 (2003) 127

[3] M.E. Gershenson, V. Podzorov, A.F. Morpurgo, Rev. Modern Phys. 78 (2006) 973.

[4] S.R. Forrest, Nature 428 (2004) 911.

[5] J.H. Burroughes, D.D.C. Bradley, A.R. Brown, R.N. Marks, K. Mackay, R.H. Friend, P.L. Burns, A.B. Holmes, Nature 347 (1990) 539

[6] J. Godlewski, M. Obarowska, Opto-Electron. Rev. 15 (2007) 179.

[7] B. Oregan, M. Grätzel, Nature 353 (1991) 737.

[8] R.F. Service, Science 306 (2004) 2034.

[9] J.E. Northrup, M.L. Chabinyc, Phys. Rev. B 68 (2003) 041202.

[10] V. Nadazdy, R. Durny, J. Puigdollers, C. Voz, S. Cheylan, K. Gmucova, Appl. Phys. Lett. 90 (2007) 092112.

[11] P.B. Miranda, D. Moses, A.J. Heeger, Phys. Rev. B 64 (2001) 081201.
[12] F.A. Hegmann, R.R. Tykwinski, K.P.H. Lui, J.E. Bullock, J.E. Anthony, Phys. Rev. Lett. 89 (2002) 227403.

[13] O. Ostroverkhova, D.G. Cooke, S. Shcherbyna, R.F. Egerton, F.A. Hegmann, R.R. Tykwinski, J.E. Anthony, Phys. Rev. B 71 (2005) 035204.

[14] V.K. Thorsmølle, R.D. Averitt, X. Chi, D.J. Hilton, D.L. Smith, A.P. Ramirez A.J. Taylor, Appl. Phys. Lett. 84 (2004) 891.

[15] O. Ostroverkhova, S. Shcherbyna, D.G. Cooke, R.F. Egerton, F.A. Hegmann, R.R. Tykwinski, S.R. Parkin, J.E. Anthony, J. Appl. Phys. 98 (2005) 033701.

[16] O. Ostroverkhova, D.G. Cooke, F.A. Hegmann, J.E. Anthony, V. Podzorov, M.E. Gershenson, O.D. Jurchescu, T.T.M. Palstra, Appl. Phys. Lett. 88 (2006) 162101.

[17] O. Ostroverkhova, J. Appl. Phys. 103 (2008) 123715.

[18] E. Hendry, J.M. Schins, L.P. Candeias, L.D.A. Siebbeles, M. Bonn, Phys. Rev. Lett. $92(2004) 196601$.

[19] G.D. Scholes, G. Rumbles, Natur. Mater. 5 (2006) 683.

[20] S.L. Dexheimer, A.D. Van Pelt, J.A. Brozik, B.I. Swanson, Phys. Rev. Lett. 84 (2000) 4425.

[21] S.V. Frolov, M. Liess, P.A. Lane, W. Gellermann, Z.V. Vardeny, M. Ozaki, K. Yoshino, Phys. Rev. Lett. 78 (1997) 4285.

[22] J.L. Bredas, D. Beljonne, V. Coropceanu, J. Cornil, Chem. Rev. 104 (2004) 4971

[23] G. Yu, J. Gao, J.C. Hummelen, F. Wudl, A.J. Heeger, Science 270 (1995) 1789.

[24] H.A. Mizes, E.M. Conwell, Phys. Rev. B 50 (1994) 11243.

[25] K. Hummer, C. Ambrosch-Draxl, Phys. Rev. B 71 (2005) 081202.

[26] C. Jundt, G. Klein, B. Sipp, J. Lemoigne, M. Joucla, A.A. Villaeys, Chem. Phys. Lett. 241 (1995) 84.

[27] The position of the energy levels of the excited states in the triplet manifold is still a matter of controversy, though it is clear that the PIA originates from T1.

[28] V.K. Thorsmølle, R.D. Averitt, J. Demsar, D.L. Smith, S. Tretiak, R.L. Martin, X. Chi, B.K. Crone, A.P. Ramirez, A.J Taylor, Phys. Rev. Lett. 102 (2009) 017401.

[29] H. Marciniak, M. Fiebig, M. Huth, S. Schiefer, B. Nickel, F. Selmaier S. Lochbrunner, Phys. Rev. Lett. 99 (2007) 176402.

[30] B. Kraabel, D. Mcbranch, N.S. Sariciftci, D. Moses, A.J. Heeger, Phys. Rev. B 50 (1994) 18543.

[31] D.V. Lang, X. Chi, T. Siegrist, A.M. Sergent, A.P. Ramirez, Phys. Rev. Lett. 93 (2001) 086802. 